\title{
AUS DEM VORWORT ZUR 4. RUSSISCHEN AUFLAGE
}

Das vorliegende Buch ist der Darstellung der Theorie des elektromagnetischen Feldes und des Gravitationsfeldes gewidmet. Dem allgemeinen Plan unserer Lehrbuchreihe ,Theoretische Physik“ entsprechend, gehen wir in diesem Band nicht auf die Elektrodynamik kontinuierlicher Medien ein, sondern beschränken uns auf die „mikroskopische“ Elektrodynamik, d. h. auf die Elektrodynamik des Vakuums und punktförmiger Ladungen.

Eine vollständige und logisch zusammenhängende Theorie des elektromagnetischen Feldes schließt die spezielle Relativitätstheorie ein, die daher der Darstellung zugrunde gelegt wird. Den Ausgangspunkt für die Ableitung der Grundgleichungen bilden die Variationsprinzipien, mit deren Hilfe man größte Allgemeinheit, Einheitlichkeit und vor allem Einfachheit in der Behandlung erreichen kann.

Die beiden letzten Kapitel enthalten eine Darstellung der Theorie des Gravitationsfeldes, d. h. der allgemeinen Relativitätstheorie. Dabei werden keine Vorkenntnisse des Lesers über Tensoranalysis vorausgesetzt, diese wird vielmehr parallel mit der Theorie entwickelt.

Für Hilfe beim Nachprüfen der Formeln und Korrekturlesendanken wir herzlich L. P. Gorkow, I. E. Dsjaloschinski und L. P. Pitajewskr.

Moskau, April 1961

L. D. LANDAU, E. M. Lifschitz

\section{VORWORT ZUR 5. RUSSISCHEN AUFLAGE}

Für die vorliegende 5. Auflage wurde das Buch völlig überarbeitet und ergänzt. Selbstverständlich sind diese Änderungen so beschaffen, daß sie den allgemeinen Aufbau und den Stil der Lehrbuchreihe nicht stören.

Eine wesentliche Abänderung (sie war von den Autoren schon seit einigen Jahren vorgesehen) besteht im Utbergang zu einer anderen vierdimensionalen Metrik im ganzen Buch, wobei von vornherein sowohl die kontravariante als auch die kovariante Schreibweise der Vierervektoren eingeführt werden mußte. Dadurch wurden eine einheitliche Bezeichnungsweise in verschiedenen Teilen dieses Buches und gleichzeitig Übereinstimmung mit dem gegenwärtig in der ganzen Physik verbreiteten System erreicht. Die Bequemlichkeit dieser Metrik ist insbesondere für weitere Anwendungen in der Quantentheorie wesentlich.

Sehr herzlich sei an dieser Stelle allen denen gedankt, die durch eine Reihe wichtiger Diskussionsbemerkungen zu diesem Buch beigetragen haben: W. L. Ginsburg, J. B. Zeldowitsch, B. J. Zeldowitsch, I. J. Kobsarew, I. D. Nowikow, W. I. Pustowort, S. I. Sirowatski und insbesondere L. P. PitaJEwSKI, mit dem ich viele Fragen erörterte, die mit der Überarbeitung dieses Buches zusammenhingen.

Mai 1966

E. M. LrFschitz 